\title{
Diagnosis of hepatocellular carcinoma: Which MRI contrast agent? Which diagnostic criteria?
}

\author{
So Yeon Kim \\ Department of Radiology, University of Ulsan College of Medicine, Seoul, Korea
}

Keywords: Carcinoma, Hepatocellular; Diagnositic imaging; Magnetic resonance imaging

See Article on Page 340

Of late, there have been substantial changes to the imagingbased diagnosis systems for hepatocellular carcinoma (HCC) in high risk patients; the Korea Liver Cancer Association-National Cancer Center (KLCA-NCC), ${ }^{1}$ the American Association for the Study of Liver Disease (AASLD), ${ }^{2}$ and the European Association for the Study of the Liver (EASL) ${ }^{3}$ all released updated guidelines for HCC in 2018. One of the most prominent changes in these latest updates is the active adoption of hepatobiliary contrast agent (HBA) for magnetic resonance imaging (MRI) of the liver, besides the conventional extracellular contrast agent (ECA). This change has fueled much recent research regarding diagnostic criteria and the proper selection of MRI contrast agent.

In the past, when ECA was the only choice, diagnostic criteria were simple and the same across the globe: arterial phase hyperenhancement and washout during the portal or delayed phases. After the introduction of HBA, the diagnostic criteria in different parts of the world took diverging paths according to regional practice patterns. The main subject of disagreement among the guidelines is the definition of "washout". The western criteria, including the Liver Imaging Reporting and Data System (LI-RADS), the main diagnostic algorithm for HCC endorsed by the AASLD, choose to restrict the timing of washout to only the portal venous phase (PVP) on HBA-MRI. However, the KLCA-NCC criteria expand the timing to the transitional phase and hepatobiliary phase (HBP) in addition to the PVP. These choices reflect different treatment strategies for HCC. ${ }^{4}$ In western countries, liver transplantation, particularly from deceased donors, is the primary option, requiring specific diagnosis to maximize organ utilization. By contrast, eastern countries prefer surgical resection and imageguided ablation whose outcomes are closely related to the early detection of HCC. Therefore, Asian countries, including Korea, tend to outweigh sensitive diagnosis over specific diagnosis. In addition to the controversy over washout, other differences among the diagnostic criteria should be noted. LI-RADS includes two additional major imaging features other than washout, threshold growth, and enhancing capsule, which are not evaluated in the KLCA-NCC 2018 and EASL criteria. Unlike the EASL criteria, which consider only hemodynamic imaging features, even on MRI, the LI-RADS and KLCA-NCC 2018 criteria take ancillary

\footnotetext{
Abbreviations:

AASLD, American Association for the Study of Liver Disease; EASL, the European Association for the Study of the Liver; ECA, extracellular contrast agent; HBA, hepatobiliary contrast agent; HBP, hepatobiliary phase; HCC, hepatocellular carcinoma; KLCA-NCC, the Korea Liver Cancer Association-National Cancer Center; LI-RADS, the Liver Imaging Reporting and Data System; LR-5, LI-RADS category 5; MRI, magnetic resonance imaging; PVP, portal venous phase
}

\section{Corresponding author : So Yeon Kim}

Department of Radiology and Research Institute of Radiology, Asan Medical Center, University of Ulsan College of Medicine, 88 Olympic-ro 43-gil, Songpa-gu, Seoul 05505, Korea

Tel: +82-2-3010-5980, Fax: +82-2-476-4719

E-mail: sykim.radiology@gmail.com

https://orcid.org/0000-0001-6853-8577 
imaging features into account to exclude typical benign lesions or other malignancy.

In this issue of Clinical and Molecular Hepatology, Lee and colleagues ${ }^{5}$ compare the diagnostic performance of LI-RADS 2018 and KLCA-NCC 2018 criteria in 273 treatment-naïve patients at high risk for HCC. Two hundred and two patients underwent HBA-MRI and 71 patients were evaluated with ECA-MRI. The authors should be congratulated for a timely and relevant comparison between HBA and ECA with application of the latest major diagnostic criteria. The authors properly frame the question regarding the impact of the diagnostic criteria on sensitivity and specificity according to the choice of contrast agent.

Lee et al. ${ }^{5}$ showed that on HBA-MRI, the KLCA-NCC 2018 criteria showed significantly better sensitivity for definite $\mathrm{HCC}$ than did LI-RADS category 5 (LR-5; $79.1 \%$ vs. $68.2 \%, P<0.001$ ), without a significant compromise in specificity. The difference in sensitivity between the two diagnostic criteria was more prominent for lesions smaller than $20 \mathrm{~mm}$ (KLCA-NCC vs. LI-RADS, 75.8\% vs. $51.6 \%)$. The improved sensitivity obtained by extending the washout to the HBP with the KLCA-NCC 2018 criteria is consistent with previous work, ${ }^{6,7}$ as the advantages of HBA-MRI mainly come from capturing the HBP signal intensity changes preceding the typical vascular profile changes during hepatic carcinogenesis. As HBA-MRI presents some drawbacks for arterial phase imaging, the current stringent washout criteria for LR-5 probably fail to obtain acceptable sensitivity. These strict washout criteria do not seem consistent with the main cause of the recent enthusiasm for HBA-enhanced sensitivity. This rather confusing approach toward washout timing in the western criteria is probably owing to a concern over a loss of specificity.

Lee et al. ${ }^{5}$ found that the specificity for definite HCC according to the KLCA-NCC 2018 criteria (93.9\%) with washout criteria extended to the HBP was not significantly different to that of LR-5 (95.4\%) with washout restricted to the PVP only. This high specificity of the KLCA-NCC 2018 criteria can be achieved by considering ancillary imaging features such as marked T2 hyperintensity or a targetoid appearance to exclude common causes of false-positive cases such as hemangiomas or non-HCC malignancy. When washout timing was expanded, studies taking such ancillary imaging features into account successfully maintained high specificity $(84.2-87.4 \%){ }_{1}^{6,7}$ while studies not considering them reported low specificity (48.4-68.1\%). ${ }^{8,9}$ However, it should be noted that the reported specificity in the study of Lee et al. ${ }^{5}$ was higher than that in previous studies. Their study may overestimate specificity as it only included surgically-proven malignancy. For example, a
non-HCC malignancy misclassified as HCC, especially with the KLCA-NCC 2018 criteria, and then treated with ablation therapy could have been excluded from the final cohort of this study. Some frequently encountered benign lesions such as eosinophilic infiltration were not listed as false-positive lesions in this study.

This study ${ }^{5}$ claimed that on ECA-MRI, the sensitivities of LR-5 and definite HCC in the KLCA-NCC 2018 criteria did not significantly differ. Nevertheless, the sensitivity of $75.8 \%$ for LR- 5 was still higher than the value of $69.4 \%$ for definite HCC in the KLCANCC 2018 criteria, although statistically not significant. This higher sensitivity for LR-5 can be attributed to the additional major imaging features that are addressed in LI-RADS 2018 but not in KLCA-NCC 2018. Lee et al. ${ }^{5}$ found that two HCCs were diagnosed as LR- 5 because of threshold growth, while these were not regarded as definite HCC according to KLCA-NCC 2018. As Lee et al. ${ }^{5}$ included only $62 \mathrm{HCCS}$ evaluated with ECA, the study might be underpowered in respect to detection of the sensitivity difference between the two diagnostic criteria. The exclusion of threshold growth and enhancing capsule from the major features for the diagnosis of definite HCC in the KLCA-NCC 2018 criteria may likely mitigate their sensitivity on ECA-MRI.

MRI contrast agents need to be selected with careful consideration of the available treatment options and liver function of patients; appropriate diagnostic criteria should be applied to maximize the unique advantages of each MRI contrast agent. When surgical resection and image-guided ablation are considered as curative options, HBA-MRI comes into its own, presenting optimal image quality in patients with preserved liver function, and survival benefits that were demonstrated in a recent large-scale study. ${ }^{10}$ Under such circumstances, diagnostic criteria should pursue sensitivity at the expense of specificity, which is a tenet of the KLCA-NCC 2018 criteria. To hold the specificity loss within an acceptable range with expanded washout timing, ancillary imaging features excluding common false positives should be incorporated. Contrarily, in a clinical setting where liver transplantation from deceased donors is considered as a primary option, ECA-MRI provides excellent specificity and its image quality is not degraded by poor liver function. When interpreting ECA-MRI, LI-RADS criteria considering threshold growth and capsular enhancement along with washout as a major diagnostic criterion may ameliorate sensitivity. The next version of the KLCA-NCC criteria is anticipated to consider more detailed features for ECA-MRI. 


\section{Conflicts of Interest}

The author has no conflicts of interests to disclose.

\section{REFERENCES}

1. Korean Liver Cancer Association (KLCA); National Cancer Center (NCC), Goyang, Korea. 2018 Korean Liver Cancer Association-National Cancer Center Korea practice guidelines for the management of hepatocellular carcinoma. Korean J Radiol 2019;20:1042-1113.

2. Marrero JA, Kulik LM, Sirlin CB, Zhu AX, Finn RS, Abecassis MM, et al. Diagnosis, staging, and management of hepatocellular carcinoma: 2018 practice guidance by the American Association for the Study of Liver Diseases. Hepatology 2018;68:723-750.

3. European Association for the Study of the Liver. EASL clinical practice guidelines: management of hepatocellular carcinoma. J Hepatol 2018;69:182-236.

4. Park JW, Chen M, Colombo M, Roberts LR, Schwartz M, Chen PJ, et al. Global patterns of hepatocellular carcinoma management from diagnosis to death: the bridge study. Liver Int 2015;35:2155-2166.

5. Lee S, Kim SS, Chang DR, Kim H, Kim MJ. Comparison of LI-RADS
2018 and KLCA-NCC 2018 for noninvasive diagnosis of hepatocellular carcinoma using magnetic resonance imaging. Clin Mol Hepatol 2020;26:340-351.

6. Kim DH, Choi SH, Kim SY, Kim MJ, Lee SS, Byun JH. Gadoxetic acidenhanced MRI of hepatocellular carcinoma: value of washout in transitional and hepatobiliary phases. Radiology 2019;292:270.

7. Joo I, Lee JM, Lee DH, Jeon JH, Han JK. Retrospective validation of a new diagnostic criterion for hepatocellular carcinoma on gadoxetic acid-enhanced MRI: can hypointensity on the hepatobiliary phase be used as an alternative to washout with the aid of ancillary features? Eur Radiol 2019;29:1724-1732.

8. Joo I, Lee JM, Lee DH, Jeon JH, Han JK, Choi BI. Noninvasive diagnosis of hepatocellular carcinoma on gadoxetic acid-enhanced MRI: can hypointensity on the hepatobiliary phase be used as an alternative to washout? Eur Radiol 2015;25:2859-2868.

9. Paisant A, Vilgrain V, Riou J, Oberti F, Sutter 0 , Laurent $V$, et al. Comparison of extracellular and hepatobiliary MR contrast agents for the diagnosis of small HCCs. J Hepatol 2020;72:937-945.

10. Kang TW, Kong SY, Kang D, Kang MW, Kim YK, Kim SH, et al. Use of gadoxetic acid-enhanced liver MRI and mortality in more than 30000 patients with hepatocellular carcinoma: a nationwide analysis. Radiology 2020;295:114-124. 